\title{
Proximal Jejunostomy With or Without Myectomy-Myotomy Modification in Five Infants With Total Intestinal Aganglionosis: An Experience With Surgical Treatments in a Single Institution
}

\author{
By Takashi Shimotake, Seitetsu Go, Hideki Tomiyama, Shigeyoshi Aoi, and Naomi Iwai \\ Kyoto, Japan
}

\begin{abstract}
Background: Total intestinal aganglionosis is characterized by the absence of intramural ganglion cells, in which the disease's involvement extends from the stomach to the anorectum. This disease was suggested previously to be incompatible with life, but recently an extended small bowel myectomy-myotomy has achieved some prolonged survivors.

Methods: Five patients with total intestinal aganglionosis underwent laparotomy at 1 to 5 days of age. Surgery was performed as a simple jejunostomy 60 to $70 \mathrm{~cm}$ below the ligament of Treitz in the initial 2, jejunustomy $30 \mathrm{~cm}$ below the ligament of Treitz in 1, and jejunostomy with myectomymyotomy modification 30 to $35 \mathrm{~cm}$ below the ligament of Treitz in the remaining 2 infants.
\end{abstract}

Results: The initial 2 patients died of sepsis, possibly derived from frequent enteritis and bacterial translocation at 7 and 8 months of age. Another patient had prolonged survival but died of hepatic failure at 1 year, 4 months. The remaining 2 children have survived beyond 2 years of age without any liver dysfunction, receiving a combination of enteral and parenteral nutrition.

Conclusions: The more proximal site $(30$ to $35 \mathrm{~cm}$ below the ligament of Treitz) of jejunostomy with myectomy-myotomy modification appeared to be preferable for prolonged survival in these 5 patients with total intestinal aganglionosis. J Pediatr Surg 37:835-839. Copyright 2002, Elsevier Science (USA). All rights reserved.

INDEX WORDS: Hirschsprung's disease, total intestinal aganglionosis, myectomy-myotomy.
$\mathbf{T}$ OTAL INTESTINAL aganglionosis is an extremely severe form of enteric nervous system maldevelopment. ${ }^{1-2}$ The aganglionosis involves the entire colon, small intestine, duodenum and stomach. ${ }^{3}$ Treatments for infants with total intestinal aganglionosis previously resulted in uniformly fatal outcomes, but recently extended myectomy-myotomy of the proximal jejunum (Ziegler) has achieved some prolonged survivors. ${ }^{4-5}$ However, the extreme rarity of the disease appears to make it difficult to assess the significance of this recently recognized surgical alternative for extensive aganglionosis without biases from differences in institutional equipment, clinical experience, or the practice of parenteral nutritional support.

Here, we describe the results of the surgical treatments for 5 such patients in a single institution.

\section{MATERIALS AND METHODS}

We treated 5 patients who had total intestinal aganglionosis between 1990 and 2000. Of the 5 children, 4 were sporadic cases and 1 had familial occurrence. In each case, the diagnosis of congenital aganglionosis was made based on histopathology, and the levels of involvement of aganglionic segments were determined by intraoperative leveling biopsies from the stomach, duodenum, jejunum, ileum, colon, and rectum.

\section{Case 1}

A male newborn baby, delivered after a 34-week gestation with a 2,100 $\mathrm{g}$ birth weight, underwent a laparotomy at the age of 1 day. His elder sister had total colonic aganglionosis and had been treated in our hospital. Intraoperative biopsy specimens all showed an absence of intramural ganglion cells from the stomach to the rectum. We made a jejunostomy $70 \mathrm{~cm}$ distal from the ligament of Treitz and treated with total parenteral nutrition (TPN). The volume of ostomy outputs remained less than $10 \mathrm{~mL}$ per day, and he repeatedly showed severe enteritis. He died of sepsis at the age of 7 months. Two years later, his younger sister was born. She had total colonic aganglionosis with extensive involvement of the ileum. She was treated uneventfully with a modified Martin's procedure.

\section{Case 2}

The second patient, delivered after a 39-week gestation with a birth weight of 2,540 g, underwent a laparotomy at the age of 3 days. Intraoperative histopathology of the gut wall biopsy specimens confirmed total intestinal aganglionosis. We performed a jejunostomy at a site $65 \mathrm{~cm}$ distal from the ligament of Treitz, and treated with TPN. The ostomy did not function, and his abdomen was persistently distended. He died of sepsis complicated with liver failure at 8 months of age.

From the Division of Surgery, Children's Research Hospital, Kyoto Prefectural University of Medicine, Kyoto, Japan.

Supported by grants from the Scientific Research Fund of the Ministry of Education, Science and Culture of Japan (Nos. 11470369 \& 13671870).

Address reprint requests to Takashi Shimotake, MD, Division of Surgery, Children's Research Hospital, Kyoto Prefectural University of Medicine, 465 Kawaramachi-Hirokoji, Kamigyo-ku, Kyoto 6020841, Japan.

Copyright 2002, Elsevier Science (USA). All rights reserved. 0022-3468/02/3706-0004\$35.00/0

doi:10.1053/jpsu.2002.32883 


\section{Case 3}

The third patient, delivered after a 41-week gestation with a 3,884 g birth weight, underwent a laparotomy at 5 days of age. Histopathology confirmed total intestinal aganglionosis during surgery. In this case, we performed a jejunostomy at $30 \mathrm{~cm}$ distal from the ligament of Treitz. His early postoperative course was favorable, and enteral feeding was started using an elemental diet $(20 \mathrm{~mL}$ of 8 times a day; Elental-P, Morinaga-Lucel Co, Tokyo, Japan). The volume of ostomy output measured between 50 and $100 \mathrm{~mL}$ per day. He gained weight up to 6,500 g until 7 months of age. However, his liver dysfunction worsened at 8 months, and he died of liver failure at 1 year, 4 months.

\section{Case 4}

The fourth patient, of 2,270 g birth weight, underwent a laparotomy at the age of 4 days. Histology findings showed a small number of ganglion cells at $3 \mathrm{~cm}$ from the ligament of Treitz; but the distal gut was completely aganglionic. A jejunostomy was made $35 \mathrm{~cm}$ below the ligament of Treitz. At 8 weeks of age, he underwent myectomymyotomy modification of a $30 \mathrm{~cm}$ proximal jejunal segment. A $10-\mathrm{cm}$ myectomy with 5-mm width starting at the histology-proven transition zone was coupled with a $20 \mathrm{~cm}$ antemesenteric myotomy of the subsequent jejunum. The depth of the myectomy/myotomy incision was kept within the submucosa, and the cut edge was spread to a width of 8 to $10 \mathrm{~mm}$. The final length of the treated jejunum was $30 \mathrm{~cm}$, and it ended as a stoma. Enteral feeding was soon started orally using breast milk and an elemental diet $(20 \mathrm{~mL}$ at 8 times a day; Elental-P, Morinaga-Lucel Co), and the volume of daily ostomy output measured between 50 and $110 \mathrm{~mL}$. Antibiotics and medications for hepatic protection were used to prevent cholestatic liver disease after the treatment for parenterally fed infants with short bowel syndrome. His growth consistently remained between the 5th and 95th percentile of the standard for weight and height. He currently is 8 years old using a combination of home parenteral nutrition and oral intake of elemental diet. He receives $60 \%$ of his calories by the enteral feeding and the other $40 \%$ from the parenteral nutritional support, respectively.

\section{Case 5}

A female newborn baby, delivered vaginally after a 39 -week gestation with a $3,698 \mathrm{~g}$ birth weight, was referred to our hospital because of abdominal distension and bilious vomitus. At laparotomy at the age of 4 days, histopathology confirmed total intestinal aganglionosis. We performed a jejunostomy $30 \mathrm{~cm}$ distal from the ligament of Treitz. On the 8th postoperative day, we performed a myectomy-myotomy for the proximal jejunal loop. A 10-cm myectomy with 5-mm width starting at the ligament of Treitz was coupled with a $20 \mathrm{~cm}$ antemesenteric myotomy. The depth of the seromuscular incision was the submucosa and the cut edge was spread to a width of $10 \mathrm{~mm}$. The treated jejunum was $30 \mathrm{~cm}$ and ended as a stoma. Her postoperative course was favorable, and enteral feeding soon was started using breast milk (20 $\mathrm{mL}$ at 8 times per day). The volume of enterostomy output remained between 40 and $100 \mathrm{~mL}$ per day. During early postoperative care, she showed no episode of enteritis, and no prophylactic antibiotics were used. She currently is in good condition at 2 years and 8 months of age using a combination of parenteral nutrition and continuing oral intake of an elemental diet (Elental-P, Morinaga-Lucel Co,). She receives $20 \%$ of her calories by oral intake and the other $80 \%$ via the parenteral route, respectively. She consistently remains within the 5th to 95th percentile of the standard for height and weight. She has not shown any signs or symptoms of liver dysfunction.

\section{RESULTS}

\section{Intraoperative Findings}

All 5 patients underwent laparotomy aged 1 to 5 days. At the initial surgery, gut caliber changes were found at a site between 90 and $110 \mathrm{~cm}$ distal from the ligament of Treitz in each case (Fig 1). The average ratio of the proximal bowel caliber to the distal one was 3.01 (Table 1). However, a histology-based transition zone was confirmed only in case 4 and was only $3 \mathrm{~cm}$ distal from the ligament of Treitz. Therefore, there was no correlation of the observed transition with the presence or absence of intramural ganglion cells in these 5 infants.

\section{Surgical Outcomes}

In cases 1 and 2, proximal enterostomy was not successful. The volume of ostomy output remained less

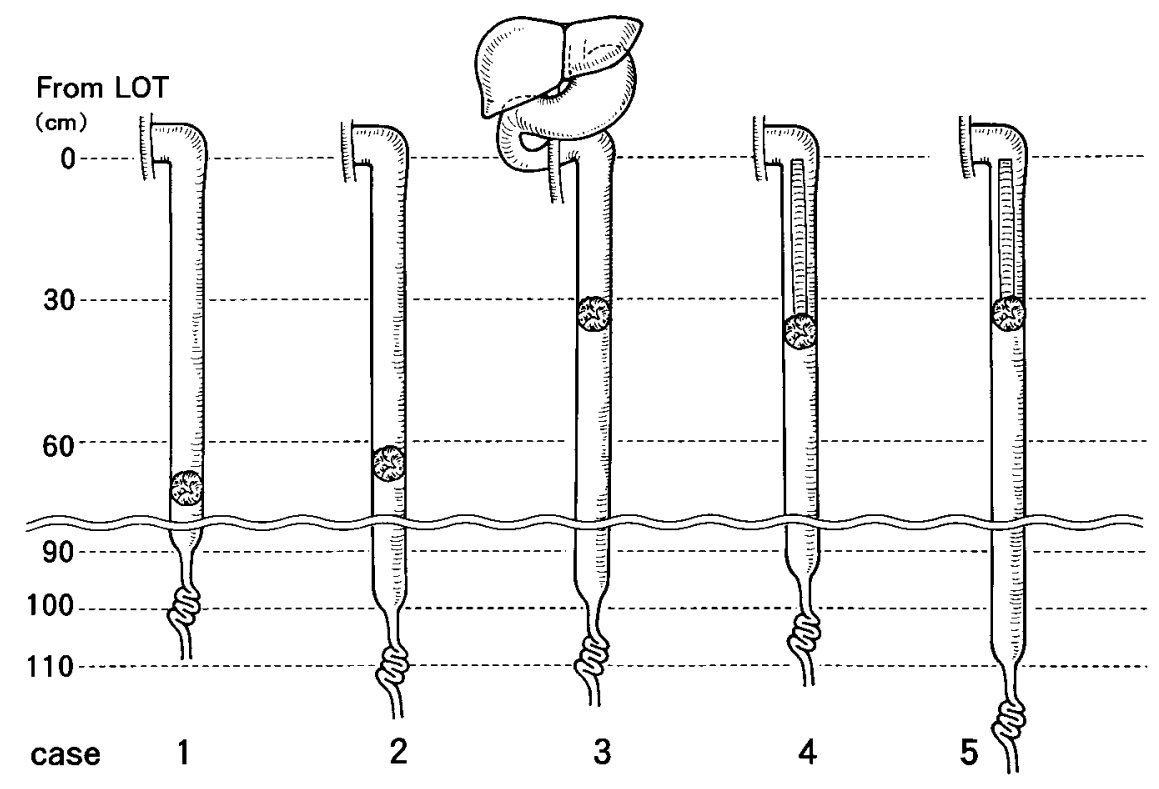

Fig 1. Schematic description of operative findings in 5 patients with total and near-total intestinal aganglionosis. Intestinal caliber changes were found at a site between 90 and $110 \mathrm{~cm}$ distal from the ligament of Treitz, but the histopathology based transition zone was confirmed only in case 4 . In the remaining 4 infants, the gastrointestinal tract was aganglionic throughout. The myectomy-myotomy procedure was performed in cases 4 and 5 at 7 weeks and 8 days of age, respectively. LOT, ligament of Treitz. 


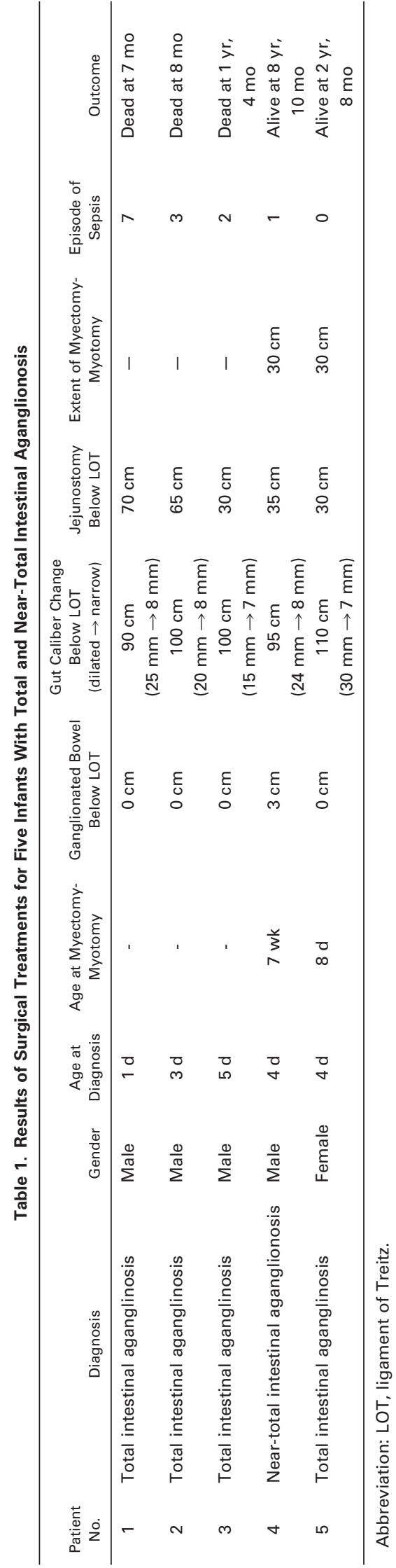

than $10 \mathrm{~mL}$ per day. They suffered from intraluminal stasis and cholestatic liver disease. Liver function measurements progressively deteriorated after repeated enteritis, and they died of sepsis at 7 and 8 months of age, respectively. In case 3, the output volume from ostomy measured between 50 and $100 \mathrm{~mL}$ per day. He grew up to the age of 7 months with enteral feeding and parenteral nutrition support. However, his liver function worsened after enteritis at 8 months of age, which led to a fatal result at 1 year, 4 months of age. The remaining 2 patients with myectomized proximal jejunal loop survived beyond 2 years of age without any liver dysfunction. They receive a combination of enteral and parenteral nutrition, in which cases 4 and 5 receive $60 \%$ and $20 \%$ of their calories by the enteral route (oral intake), respectively. Both of them remain within the 5th to 95 th percentile of the standard for height and weight after the age of 1 year (Fig 2).

\section{DISCUSSION}

The majority of enteric ganglion cells are derived from the neural crest and migrate into the intestinal wall in a caudal direction between the 5th and 12th gestational weeks. ${ }^{6,7}$ Total intestinal aganglionosis is a rare condition in which the neural crest-derived cell development is disrupted severely. ${ }^{8-10}$ This type of congenital aganglionosis is estimated to occur in less than $0.1 \%$ of patients with Hirschsprung's disease. ${ }^{11,12}$ The extreme rarity of the disease makes it difficult to assess the detailed pathophysiologic conditions. The pathology of total intestinal aganglionosis may be different from that of near-total intestinal aganglionosis, in which the ganglionated bowel often extends distally from the ligament of Treitz. ${ }^{13}$ In total intestinal aganglionosis, a lack of peristaltic propulsion by the ganglionated bowel theoretically is expected. ${ }^{14}$ In such pathologic conditions, the intraluminal stasis of the gastric, bile, and pancreatic juice may be harmful to the sphincter function of the papilla of Vater and the surrounding region resulting in severe cholestatic liver injury or dysfunction. An extended myectomy-myotomy for the aganglionic proximal small bowel produced encouraging results in children with both total and near-total intestinal aganglionosis. ${ }^{5}$ However, the questions of whether and how surgery for total intestinal aganglionosis should be modified from that for near-total intestinal aganglionosis have not been answered clearly.

Here we described the results of surgical treatment for 5 infants with total or near-total intestinal aganglionosis, 4 of whom had no ganglionated bowel below the ligament of Treitz (Table 1). In the initial 2 patients, we performed a jejunostomy 60 to $70 \mathrm{~cm}$ distal from the ligament of Treitz during the neonatal period, but could 


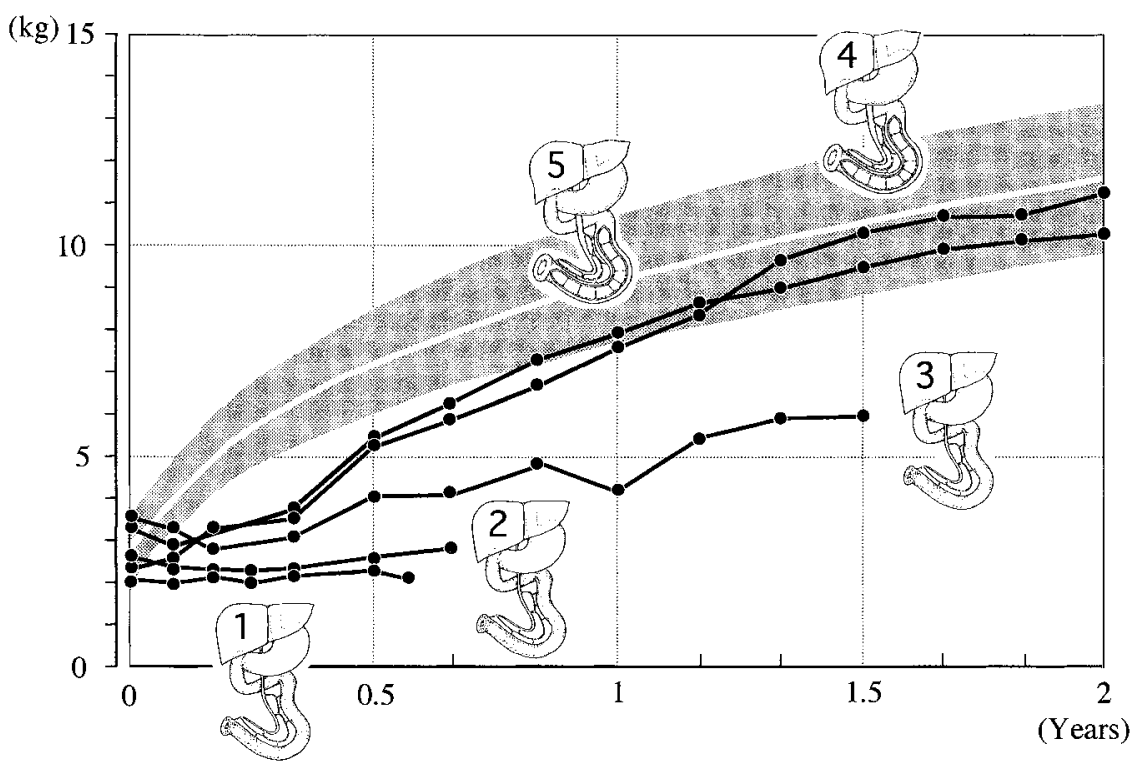

Fig 2. Postoperative body weight of 5 infants with total and near-total intestinal aganglionosis. Cases 1 and 2 suffered from enteritis and intraluminal stasis and died of sepsis at 7 and 8 months of age, respectively. Patient 3 achieved prolonged survival, but died of hepatic failure at 1 year, 4 months. Cases 4 and 5 , with the jejunal segment myectomized, have survived without any liver dysfunction, receiving a combination of enteral and parenteral nutrition. The shaded area indicates the range of the 5th to 95th percentile of age-matched infants. perform no additional myectomy-myotomy surgical intervention because of their deteriorating general condition. Severe enteritis and intraluminal stasis in the proximal jejunum could not be controlled because the proximal jejunal loop was too long to be effectively irrigated and also because antibiotics could not relieve the overwhelming bacterial overgrowth. In previous studies, a jejunostomy with myectomy-myotomy modification 50 to $70 \mathrm{~cm}$ below the ligament of Treitz was reported to function in several cases with near-total intestinal aganglionosis. ${ }^{13,15}$ However, we performed it 30 to $35 \mathrm{~cm}$ below the ligament of Treitz in the subsequent 3 infants because a jejunostomy 60 to $70 \mathrm{~cm}$ below the ligament of Treitz appeared to us excessively long for total intestinal aganglionosis. Theoretically, myectomy can relieve the resistance to the entry of luminal contents into that segment of the intestine, but the resistance is never zero and will cause obstruction if the segment is too long. ${ }^{13}$ At an aganglionic segment with a length greater than 20 to $40 \mathrm{~cm}$, the small bowel passive conduit length would be too long to permit proximal propulsive forces to push the luminal contents through, resulting in a functional obstruction and intraluminal stasis. ${ }^{5}$

In these 5 infants, we found a gut caliber change at a site between 90 and $110 \mathrm{~cm}$ distal from the ligament of Treitz. However, the histologically proven transition was confirmed only in case 4 . There was no correlation of the observed transition with the distribution of enteric ganglia in these patients. Based on most previous descriptions, the presence of gut caliber change may be inexplicable in the totally aganglionic gastrointestinal tract. This unique phenomenon may be derived from a passive fluid retention in the proximal gut during embryogenesis, which was aggravated from fetal amniotic fluid ingestion and functional obstruction of the distal gut. Attention should be paid to possible discrepancies between macroscopic and microscopic transition zones because the presence of a gut caliber change does not always indicate a location for which an ostomy should be made in this rare disease.

The absorptive capability of the myectomized aganglionic bowel remains to be assessed in children with total and near-total intestinal aganglionosis. In cases 3 through 5, we decided to perform a jejunostomy 30 to 35 $\mathrm{cm}$ distal from the ligament of Treitz for patients with total intestinal aganglionosis after the difficult clinical course in the initial 2 cases. A more proximal jejunostomy, as performed in the current patients, may be advantageous for the control of enteritis and intraluminal stasis, but may lead to poor absorptive activity. ${ }^{16,17}$ In cases 4 and 5, who had a 30- to $35-\mathrm{cm}$ jejunal segment myectomized, body weight and height both remain between the 5th and 95th percentile of the standardized curve (Fig 2). However, they can receive only $20 \%$ to $60 \%$ of their calories by oral feeding, and the parenteral nutrition support has not been discontinued. Considering the findings reported on infants with short bowel syndrome, a 30- to 35-cm proximal jejunal loop must be too short eventually to support adequate enteral absorption to predict a TPN-free related survival. ${ }^{15,17}$ Alternatively, a second myectomized $30 \mathrm{~cm}$ of more distal jejunoileum with a second stoma, which could be fed, may be a feasible and attractive option for such patients. ${ }^{12}$ Pursuit of the potential to be fully nourished enterally in infants with total intestinal aganglionosis remains a challenge for pediatric surgeons. ${ }^{18,19}$ Intestinal absorption/adapta- 
tion of the aganglionic bowel segment, the patient's growth, and possible TPN-associated liver injury should be investigated further based on a greater number of children with total intestinal aganglionosis. ${ }^{20,21}$ How- ever, considering that the outlook for gut nutritional absorption is hardly optimistic in many patients with total intestinal aganglionosis, this disease entity remains a candidate for intestinal transplantation.

\section{REFERENCES}

1. Caniano DA, Ormsbee HS III, Polito W, et al: Total intestinal aganglionosis. J Pediatr Surg 20:456-460, 1985

2. MacKinnon AE, Cohen SJ: Total intestinal aganglionosis, an autosomal recessive condition? Arch Dis Child 52:898-899, 1977

3. Di Lorenzo M, Yazbeck S, Brochu P: Aganglionosis of the entire bowel: Four new cases and review of the literature. Br J Surg 72:657658,1985

4. Talwalker VC: Aganglionosis of the entire bowel. J Pediatr Surg 11:213-216, 1976

5. Ziegler MM, Royal RE, Brandt J, et al: Extended myectomymyotomy a therapeutic alternative for total intestinal aganglionosis. Ann Surg 218:504-511, 1993

6. Okamoto E, Ueda T: Embryogenesis of intramural ganglia of the gut and its relation to Hirschsprung's disease. J Pediatr Surg 2:437-443, 1967

7. Jacob-Cohen RJ, Payette RF, Gershon MD, et al: Inability of neural crest cells to colonize the presumptive aganglionic bowel of ls/ls mutant mice: Requirement for a permissive microenvironment. J Comp Neurol 255:425-438, 1987

8. Schuchardt A, D'Agati V, Larsson-Blomberg L, et al: Defects in the kidney and enteric nervous system of mice lacking the tyrosine kinase receptor Ret. Nature 367:380-383, 1994

9. Inoue $\mathrm{K}$, Shimotake $\mathrm{T}$, Iwai $\mathrm{N}$ : Mutational analysis of RET/ GDNF/NTN genes in children with total colonic aganglionosis with small bowel involvement. Am J Med Genet 93:278-284, 2000

10. Chalazonitis A, Pham TD, Rothman TP, et al: Neurotrophin-3 is required for the survival-differentiation of subsets of developing enteric neurons. J Neurosci 21:5620-5636, 2001
11. Martin LW: Surgical management of Hirschsprung's disease involving small intestine. Arch Surg 97:183-189, 1968

12. Attie T, Pelet A, Edery P, et al: Diversity of RET protooncogene mutations in familial and sporadic Hirschsprung's disease. Hum Mol Genet 4:1381-1386, 1995

13. Zerella JT, Ingebo KR: A 3-year survivor of near-total intestinal aganglionosis. J Pediatr Surg 28:1589-1591, 1993

14. Nemeth L, Yoneda A, Kader M, et al: Three-dimensional morphology of gut innervation in total intestinal aganglionosis using whole-mount preparation. J Pediatr Surg 36:291-295, 2001

15. Ziegler MM, Ross AJ, Bishop HC: Total intestinal aganglionosis: A new technique for prolonged survival. J Pediatr Surg 22:82-83, 1987

16. Andorsky DJ, Lund DP, Lillehei CW, et al: Nutritional and other postoperative management of neonates with short bowel syndrome correlates with clinical outcomes. J Pediatr 139:27-33, 2001

17. Georgeson KE, Breaux CW Jr: Outcome and intestinal adaptation in neonatal short-bowel syndrome. J Pediatr Surg 27:344-350, 1992

18. Bianchi A: Intestinal loop lengthening-A technique for increasing small intestinal length. J Pediatr Surg 15:145-151, 1980

19. Coran AG, Teitelbaum DH: Recent advances in the management of Hirschsprung's disease. Am J Surg 180:382-387, 2000

20. Moss RL, Das JB, Raffensperger JG: Total pareteral nutritionassociated cholestasis: Clinical and histopathologic correlation. J Pediatr Surg 28:1270-1275, 1993

21. Farmer DG, McDiarmid SV, Yersiz H, et al: Outcome after intestinal transplantation: Results from one center's 9-year experience. Arch Surg 136:1027-1031, 2001 